\title{
Route Optimization for Proxy Mobile IPv6 in IMS Network
}

\author{
Tsunehiko Chiba, Hidetoshi Yokota \\ KDDI R\&D Laboratories, Inc. \\ Saitama, Japan
}

\author{
Ashutosh Dutta, Dana Chee \\ Telcordia Technologies, Inc. \\ New Jersey, USA
}

\author{
Henning Schulzrinne \\ Columbia University, \\ New York, USA
}

\begin{abstract}
Localized mobility protocols are designed to address many of the drawbacks such as additional signaling and over-the-air tunnel overhead associated with global mobility protocols like Mobile IPv4 and Mobile IPv6. Proxy Mobile IPv6 is one such network controlled localized protocol defined in the IETF. Although Proxy Mobile IPv6 can resolve longer binding update and tunnel overhead related issues when a mobile node's movement is confined to a specific domain, some of the handoff-related functions and data paths between two communicating nodes still need to be optimized. The data path reduction between the communicating nodes helps to reduce one way packet delay when both nodes are under the same localized domain and the local mobility anchor point is away. The process of reducing the data path is often referred to as route optimization. Route optimization helps to reduce the delay due to media delivery that is critical for real-time application. We propose several route optimization techniques that can be applied to Proxy Mobile IPv6 to improve the efficiency of media delivery for both intra- and inter-domain movements. We select one of these proposed route optimization mechanisms and then describe the implementation details and analyze the experimental results.
\end{abstract}

Keywords; Mobility protocols, Proxy Mobile IP, Route optimization

\section{INTRODUCTION}

Wireless network providers strive to provide secure and seamless connectivity to end users. When an MN (Mobile Node) is subjected to repeated handoff, the quality of the existing communication is degraded due to delay in packet delivery and packet loss. There are several transition events during the handoff, including network discovery, configuration, authentication, security association, binding update and media redirection. Each of these events contributes to the handoff delay and the associated packet loss. Several optimization techniques have been proposed at all layers to reduce the handoff delay associated with each event. On the other hand, a route optimization technique is required in order to reduce the delay due to media delivery by limiting the media traversal path between the communicating nodes. In order to reduce the delay due to binding update and associated one-way-delay of media delivery, various local mobility management protocols such as HAWAII [1], Cellular IP [2] and HMIPv6 [3] have been designed. These protocols are preferably used when an MN's movement is confined to a specific domain. The NETLMM working group within the IETF has recommended a set of goals and host requirements to support localized mobility [4] [5]. Based on these goals, the IETF defines Proxy Mobile
IPv6 (PMIPv6) protocol [6] that helps to reduce the delay due to longer binding update. PMIPv6 also relieves the MN of much of the burden and transfers it to the wired access routers within the network that are called MAGs (Mobility Access Gateways). A MAG sends a PBU (Proxy Binding Update) message to the LMA (Local Mobility Anchor), which acts like an HA (Home Agent) in Mobile IPv6 (MIPv6) [7], on behalf of the MNs. Each MN is anchored with a certain MAG until it performs handoff to a new MAG. Thus, PMIPv6 is preferred when mobility is confined within a domain and wireless network providers can avoid overloading the MNs by setting up a tunnel between the MN and the LMA. In general, a tunneling mechanism is not desirable for the $\mathrm{MN}$ because it adds extra processing and bandwidth constraints to the wireless hop. Although PMIPv6 is an improvement over existing global mobility protocols, it still needs to be enhanced in certain areas such as route optimization. Route optimization is the process of reducing the path between two communicating nodes. Route optimization for PMIPv6 is intended to further reduce delay due to media delivery between the two communicating nodes by reducing the traversal distance of the media packets. In particular, when the communicating nodes are outside the home network and are in the visited network, and the LMA is situated far from the visited networks, there is still an opportunity to optimize the route by avoiding paths via the local LMA. In this paper, we propose several mechanisms that can provide route optimization in a PMIPv6 environment. We analyze each of the optimization techniques and describe the associated pros and cons. These route optimization techniques are most suitably deployed when both the MNs are in the same visited domain.

The remainder of the paper is organized as follows. We provide an overview of PMIPv6 in Section II. Section III describes the related work that supports route optimization in PMIPv6. Section IV describes the proposed route optimization techniques and the pros and cons of each of these techniques. Analytical results and experimental results from the implementation are described in Section V. Finally, Section VI concludes the paper.

\section{OVERVIEW OF PMIPV6}

PMIPv6 has several functional components that interact with each other while providing the desired mobility functions. PMIPv6 does not need any mobility stack on the MN but rather 
uses the mobility proxies on the MAG to help perform the required mobility binding operations.

In a PMIPv6 environment, when the $\mathrm{MN}$ is outside the home network and moves within the same LMA domain or between two LMA domains in the visited network, the MN is always configured with the home address. The MAG helps the MN to authenticate with the AAA (Authentication, Authorization and Accounting) server and then sends the appropriate IPv6 prefix as part of the router advertisement for stateless auto-configuration, or the MAG can also act as a DHCP relay agent for stateful auto-configuration. Figure 1 shows the network elements associated with PMIPv6 operation. MAG\#1 and MAG\#2 are the proxy mobility agents in visited network 1 and visited network 2, respectively. The MN connects to the new MAG (MAG\#2) after its movement to a new domain. During the network attachment procedure, MAG\#2 communicates with the AAA and verifies the MN's profile and then sends a PBU message to the LMA. The LMA delivers the home link prefix to the MAG\#2 as part of the PBA (Proxy Binding Acknowledgement) message. At this time, a bidirectional tunnel is established between the LMA and MAG\#2 and the home link prefix is sent over the router advertisement message. The MN configures its address based on the home link prefix in the router advertisement message. In this example, the CN (Corresponding Node) can be far away, or it can be situated in either of the visited networks. When both the MN and $\mathrm{CN}$ are situated in the visited networks, and the visited networks are adjacent to each other, media traversal via the LMA can be optimized. Proposed route optimization techniques help mitigate the media path by avoiding routing data via the LMA.

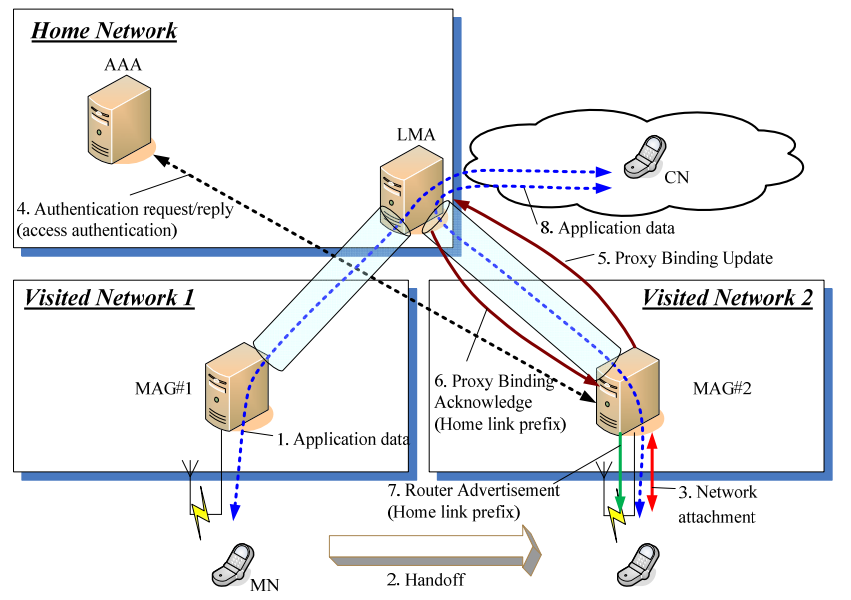

Figure 1: Overview of PMIPv6 operation

\section{RELATED WORK}

Route optimization work has been carried out for many mobility protocols. For example, there was a proposal to extend Mobile IPv4 [8] to support route optimization, however, it never got standardized; MIPv6 provides an in-built route optimization mechanism so that data does not take a longer route via the HA and is instead sent directly to the $\mathrm{CN}$.
Similarly, route optimization of SIP (Session Initiation Protocol) signaling has been described in [9]. Some of the general requirements to provide route optimization in a PMIPv6 environment are mentioned in [10]. Based on these requirements, there are also a few proposals that have discussed route optimization for PMIPv6 [11] [12]. These mechanisms either depend on the MIPv6 route optimization procedures or introduce a heavyweight route setup procedure to obtain the desired optimization. Sarikaya et al. [11] use an enhanced route optimization technique where the MAG acts on behalf of the MN and uses a cryptographically generated home address so that no home test is needed after the initial home test. It discusses scenarios involving intra- and inter-domain movement. However, there is no clear explanation of how route optimization is supported at the CN. The MAG is dependent on a policy to find out if the CN supports route optimization or not. Abeille et al. [12] provide efficient setup and maintenance for a route optimized path between the MN and the MAG, and between the $\mathrm{CN}$ and the MAG. However, it introduces an additional element called a route optimization controller. It also proposes an additional set of messages that might add complexity to the system and overhead on the air. Koodli et al. [13] propose techniques for localized routing for intra- and inter-MAG scenarios. However, it depends upon a third party service such as a proxy to determine the location of the MNs. In this paper, we propose a set of lightweight route optimization techniques for PMIPv6 that can be applied to both intra- and inter-LMA types of handoff. These techniques take advantage of the data packet in the session and set up the route optimization between the $\mathrm{MN}$ and $\mathrm{CN}$.

\section{Proposed Mechanism For Route Optimization}

In this section, we introduce two scenarios that illustrate how the route optimization technique can be applied to PMIPv6 and discuss these techniques in details. We then describe four alternative approaches for realizing inter-MAG route optimization that can support both intra- and inter-LMA route optimizations.

\section{Intra-LMA Route Optimization:}

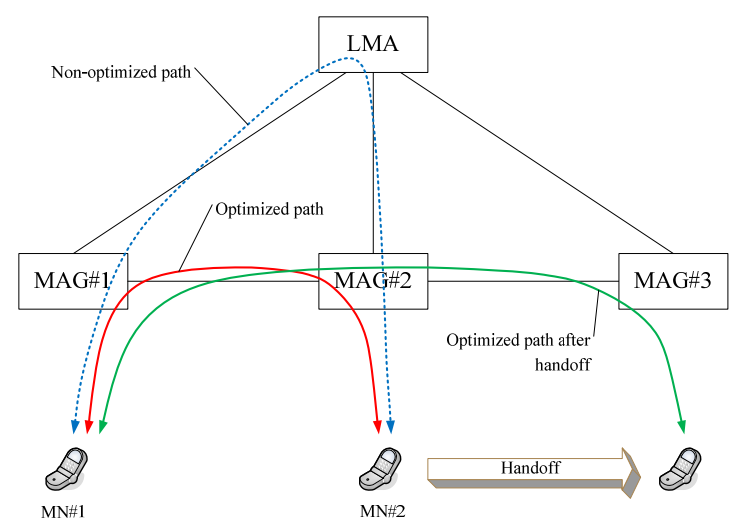

Figure 2: PMIPv6 route optimization (intra-LMA scenario) 
Figure 2 shows the basic network configuration of PMIPv6 architecture that involves an MN's intra-LMA movement. MN\#1 and MN\#2 could belong to the same PMIPv6 domain or different ones. If the LMA is placed too far from the MAG, then media delivery between $\mathrm{MN \# 1}$ and $\mathrm{MN \# 2}$ will be considerably delayed. In this specific intra-LMA scenario, MN\#1 is anchored at MAG\#1 and MN\#2 is anchored at MAG\#2. MN\#2 establishes communication with MN\#1 and then performs handoff to MAG\#3. Without any route optimization, before the handoff, data communication between MN\#1 and MN\#2 goes via MAG\#1, LMA, and MAG\#2 and after the handoff it goes via MAG\#3. Thus, it is desirable to reduce the route path associated with data traversal before and after handoff. For intra-LMA scenario, MN\#1 and MN\#2 operate under the same LMA and the MN's movement is confined to the MAGs that are under the same LMA. When the route optimization technique is deployed, the communication path is shortened as the data bypass the LMA. The dotted line shows the non-optimized path through the LMA and the solid lines show the route optimized path that bypasses the LMA.

\section{Inter-LMA Route Optimization:}

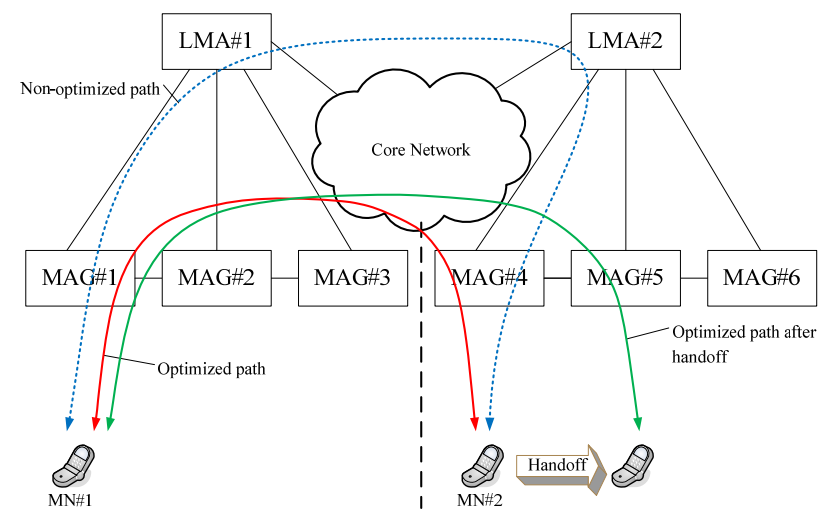

Figure 3: PMIPv6 route optimization (inter-LMA scenario)

Figure 3 shows the scenario depicting the inter-LMA route optimization. In Figure 3, MN\#1 and MN\#2 belong to two different PMIPv6 domains and thus are under the control of two different LMAs. MN\#1 is under MAG\#1 that is under LMA\#1 and MN\#2 is under MAG\#4 that is under LMA\#2. After the initial attachment, MN\#2 moves to MAG\#5, which is also under LMA\#2. Without any route optimization technique, the media flows via LMA\#1 and LMA\#2. The route optimization technique helps to shorten the data traversal path by bypassing LMA\#1 and LMA\#2. The dotted line in Figure 3 shows the non-optimized path through LMAs, whereas the solid lines show the route optimized path that bypasses LMA\#1 and LMA\#2.

\section{A. Approach 1: Binding Cache Entry at LMA and MAG}

Figure 4 shows the basic optimization procedure and flows associated with one of the optimization techniques, which utilizes BCE (Binding Cache Entry) at the LMA and at the MAG. In Figure 4, we first show the path optimization from MN\#1 to MN\#2. Before the handoff, MN\#1 attaches to MAG\#1 and then MAG\#1 sends a PBU message to the LMA on behalf of MN\#1. Similarly, MN\#2 connects to MAG\#2, which triggers a PBU message to the LMA on behalf of MN\#2. The initial packet from MN\#1 to MN\#2 is tunneled and is sent via the LMA. As soon as the LMA gets this packet, it figures out how to forward the packet to MAG\#2. Then, the LMA sends a new message called CBU (Correspondent Binding Update) message to MAG\#1 notifying that MAG\#2 is the anchoring node for MN\#2. After getting this CBU message, MAG\#1 keeps a cache that maps MAG\#2 with MN\#2 and sends a response message called CBA (Correspondent Binding Acknowledge) message to the LMA. Thus, any subsequent packet from MN\#1 destined for MN\#2 gets intercepted by MAG\#1 and is forwarded to MAG\#2, instead of being forwarded to the LMA. The trajectory of the route optimized packet thus becomes: MN\#1->MAG\#1->MAG\#2->MN\#2 instead of MN\#1->MAG\#1->LMA->MAG\#2->MN\#2, thereby optimizing the route of the data packet from $\mathrm{MN \# 1}$ to MN\#2.

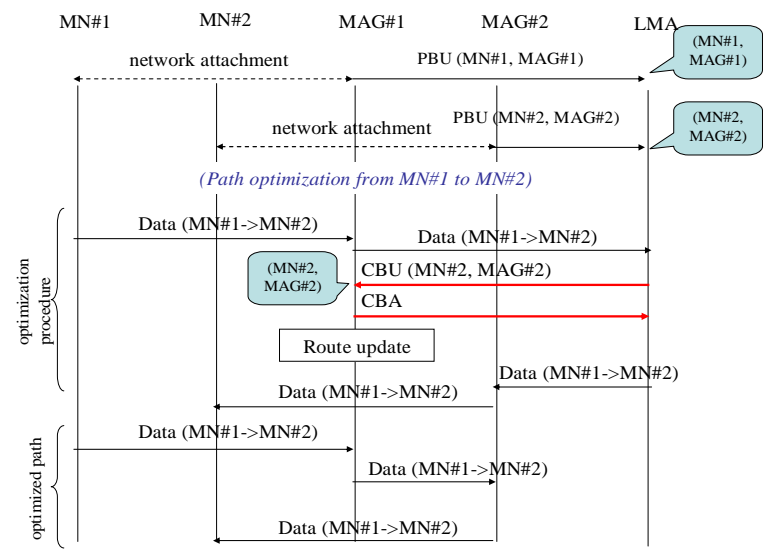

Figure 4: BCE-based optimization

(data traversal MN\#1->MN\#2)

Similarly, Figure 5 shows the optimized data path from MN\#2 to MN\#1 using the BCE-based approach. In this specific case, the LMA actually sends the CBU message to MAG\#2 with the parameters of MN\#1 and MAG\#1 during the optimization procedure. Thus, the data path from MN\#2 destined to MN\#1 is optimized. 


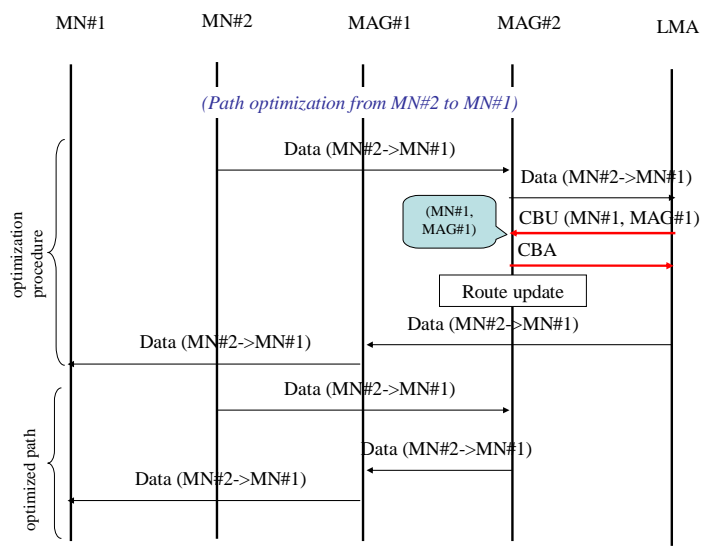

Figure 5: BCE-based optimization (data traversal MN\#2->MN\#1)

Figure 6 shows the route optimization procedure and optimized route from MN\#1 to MN\#2 after MN\#2 performs handoff to a new MAG (MAG\#3). In this case, MN\#2 attaches to MAG\#3 and sends a PBU message to the LMA. At that point, the LMA immediately sends a CBU message to MAG\#2, since the LMA is aware of the presence of MAG\#2. MAG\#2 in turn sends a CBU message to MAG\#1 notifying that MN\#2 is under MAG\#3. Thus, both MAG\#1 and MAG\#2 are made aware of the fact that MN\#2 is currently connected to MAG\#3. Similarly, data from MN\#2 to MN\#1 can be optimized after MN\#1 has performed handoff. For the sake of brevity, we do not show this procedure. During this procedure, if any data from MN\#1 destined for MN\#2 arrives at MAG\#1, it forwards the data to MAG\#2, which in turn forwards it to MAG\#3. MAG\#3 finally forwards it to MN\#2. However, once the route optimization procedure is over, any data destined from MN\#1 to MN\#2 gets intercepted by MAG\#1 and then gets forwarded directly to MAG\#3 which forwards it again to MN\#2.



Figure 6: BCE-based optimization after handoff
In Figure 7, we show a BCE-based route optimization technique to support the inter-LMA scenario. For simplicity, we describe the data path from MN\#1 to MN\#2 only. In this scenario, MN\#1 and MN\#2 belong to MAGs associated with two different LMAs. When LMA\#2 receives a packet from MN\#1 to MN\#2, LMA\#1 sends a CBU message to LMA\#1 and LMA\#1 also sends it to MAG\#1 to notify the route update.



Figure 7: BCE-based optimization for inter-LMA

While the BCE-based mechanism causes less signaling overhead compared to other approaches, the path for the first packet is not optimized and is subjected to added delay.

\section{B. Approach 2: Binding Query}

The second approach uses a binding query mechanism whereby a packet, instead of getting routed to the LMA, gets diverted to the appropriate MAG meant for the destination node. Thus, unlike the previous approach, the very first packet is also route optimized at the expense of additional look-up delay at the MAG. Figure 8 shows an example where the binding query-based approach is used to optimize the data path.

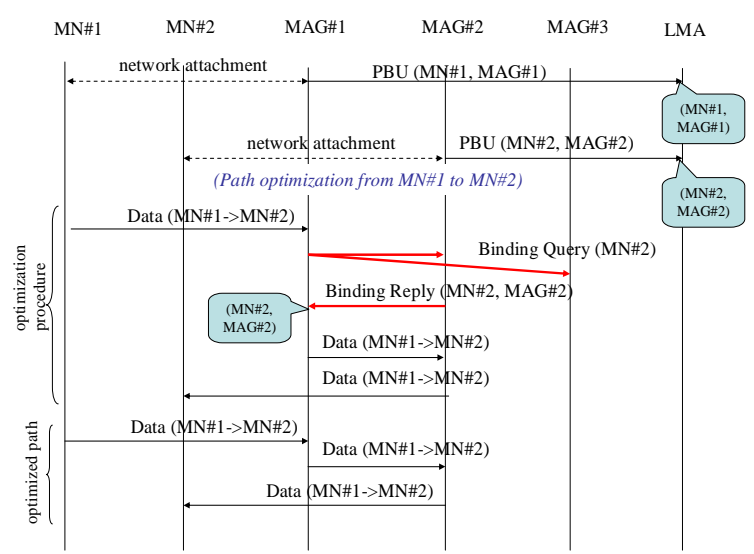

Figure 8: Binding query-based optimization (data traversal MN\#1->MN\#2) 
In Figure 8, we show how MAG\#1 can query the neighboring MAGs to find out if a specific target $\mathrm{MN}$ is anchored at one of these MAGs. In this scenario, when a packet from MN\#1 to MN\#2 is intercepted by MAG\#1, MAG\#1 sends a binding query message to both MAG\#2 and MAG\#3, which are the neighboring MAGs. Since MN\#2 is anchored at MAG\#2, it sends a binding reply message including the addresses of MN\#2 and MAG\#2. Thus, MAG\#1 can directly send the data to MAG\#2 instead of forwarding it to the LMA. There may be a short delay for the first packet because of the associated binding query message and binding reply message. However, for any subsequent data destined for MN\#2, MAG\#1 does not need to send any query, but rather looks up its cache and forwards the data to MAG\#2 that in turn delivers it to MN\#2. MAG\#1 can either unicast this query to a list of MAGs or multicast the query using a localized scope-based multicast address. A similar procedure will take place for the packets from MN\#2 to MN\#1.

Compared to the first approach, the first packet is also route optimized in this approach. However, it introduces delay due to route look-up by inspecting the packet at the MAG and there is more signaling between the MAGs to check the BCEs. One way to reduce the signaling delay between the neighboring MAGs is to transmit the signaling messages using localized multicasting. We describe the multicast-based binding cache approach as the next alternative approach.

\section{Approach 3: Multicasting binding cache to MAGs}

As each of the MNs gets anchored with a MAG, each MAG sends PBU messages to the LMA. Thus, at any point in time, the LMA maintains the BCE with a mapping of the MN and the corresponding MAG. Since the LMA is in the local domain, it can always multicast its binding cache to all the MAGs that act as anchors for the corresponding MNs. It can use localized scope-based multicast to communicate with the MAGs that are under its respective domain. This way, the neighboring MAGs always have the forwarding cache entry ahead of time. Thus, when a data packet from MN\#1 to MN\#2 arrives at MAG\#1, it can look up the local cache and forward the traffic to the corresponding anchor MAG (MAG\#2). Figure 9 shows such an example. In this case, the first data packet also gets routed through the optimized path since the MAGs have been updated during the PBU messages that have been multicast to the MAGs. If more MNs connect to the MAGs, then the BCE at the LMA gets updated, which in turn updates the MAGs by way of scope-based localized multicasting. For brevity, the data path from MN\#2 to MN\#1 is not shown here.

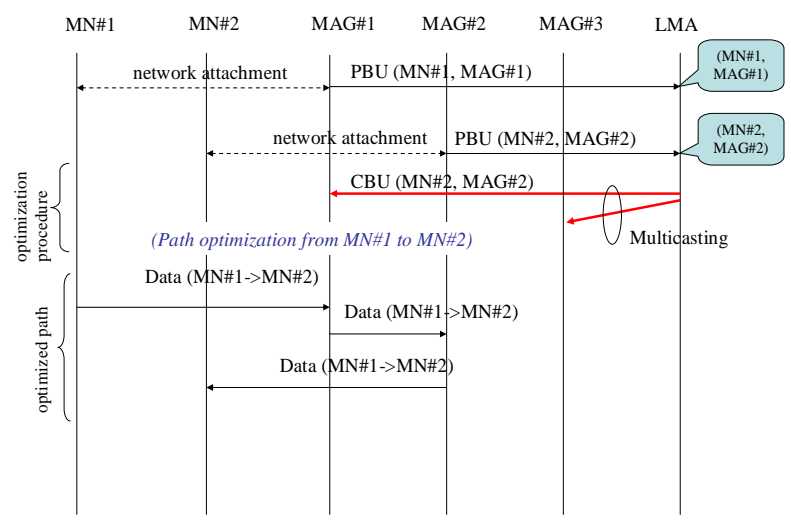

Figure 9: Binding multicast-based optimization (data traversal MN\#1->MN\#2)

\section{Approach 4: Ring, bus and mesh-based optimization}

This solution assumes that the data destined from one MN to another MN flows through each of the MAGs before it actually reaches the LMA. Depending upon the anchor meant for a specific destination MN, the data get dropped at that specific MAG (MAG\#1). Figure 10 shows a possible network configuration that can provide PMIPv6 route optimization. The MAGs and LMA can be configured in various ways, such as ring, bus and mesh topology as shown in Figure 10. However, we focus on just the ring topology here. In the case of ring-based topology, the data traffic from MN\#1 to MN\#2 or MN\#2 to MN\#1 can take advantage of an add/drop technique [14] when the data packet travels via the ring.

Figure 11 shows how the data flows via each of the MAGs and the LMA that are connected in a ring fashion, and how the data destined to MN\#1 gets dropped at the anchor MAG meant for that MN. For example, data destined for MN\#2 get dropped at MAG\#2, which then forwards it to MN\#2 without getting forwarded to the LMA. Similarly, after MN\#2 performs handoff to MAG\#3, MAG\#3 becomes the anchor for MN\#2. Thus, any data destined for MAG\#3 now gets dropped at MAG\#3 without being forwarded to the LMA. MAG\#3 forwards this packet to MN\#2, as it is the current anchor for MN\#2. This approach provides numerous benefits such as no additional signaling traffic among MAGs, no signaling traffic during the MN's handoff and added scalability by reducing the number of tunnels. However, this approach needs all the data to traverse via all the MAGs, and may consequently need a high-speed backbone network. 




Ring

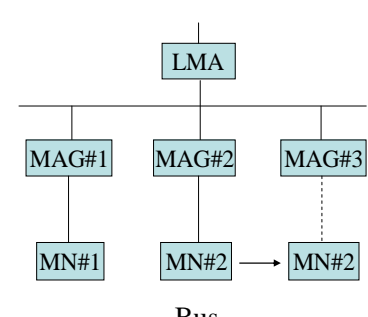

Bus



Full mesh

Figure 10: Ring, bus and mesh-based approach

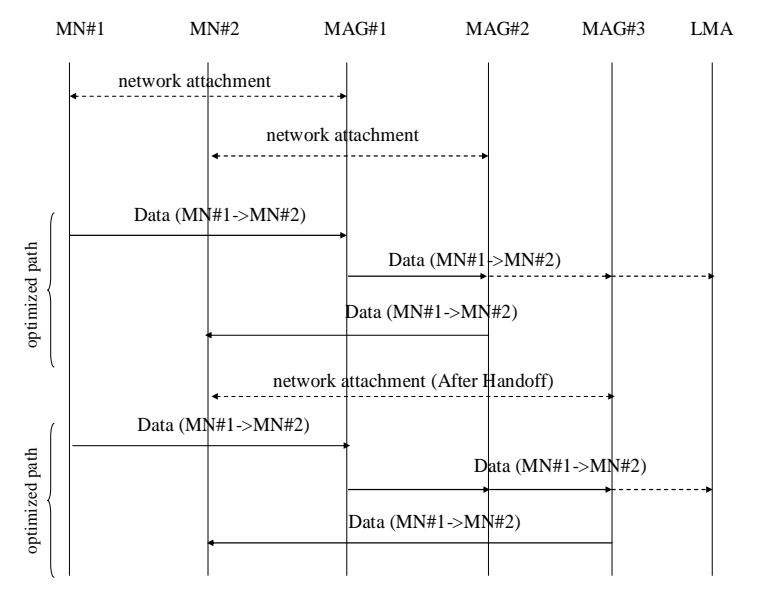

Figure 11: Ring-based optimization (data traversal MN\#1->MN\#2)

\section{QUANTITATIVE AND EXPERIMENTAL RESUlTS}

Route optimization clearly offers an advantage in a scenario where both MNs are in the visited network under one LMA. First, we provide a quantitative analysis using this route optimization technique. Then we provide the experimental results comparing route optimization and non-route optimization.

\section{A. Quantitative Analysis}

Ki-Sik Kong et al. [15] have performed a comparative analysis of handoff latency for PMIPv6 and MIPv6. However, they do not provide any analytical results comparing route optimization and non-route optimization methods. We perform an analytical comparison between route optimization for PMIPv6 and non-route optimization. We therefore define the following parameters for performance comparison. $t_{\text {map }}$ : Over-the-air packet traversal delay between the MN and AP (Access Point)

$t_{a r}$ : Packet traversal delay between the AP and MAG

$t_{m l}$ : Packet traversal delay between the MAG and LMA

$t_{m 12}$ : Inter-MAG packet traversal delay between MAG\#1 and MAG\#2

$t_{\text {tunnel: }}$ Processing delay due to tunneling and de-tunneling operations at MAG and LMA

Assuming that the MAGs are not adjacent to each other, a tunnel needs to be set up between MAG\#1 and MAG\#2 to route the packets. On the other hand, when the packet traverses via the LMA, the tunneling and de-tunneling operations take place twice, once between MAG\#1 and the LMA, and the second one between MAG\#2 and the LMA. Thus, in the case of non-route optimization, the time taken for a packet to travel from $\mathrm{MN \# 1}$ to $\mathrm{MN \# 2}$ can be represented as follows:

$$
T_{\text {non-ro }}=2 t_{\text {map }}+2 t_{\text {ar }}+t_{m 1}+t_{\text {tunnel }}
$$

In the case of route optimization, the time taken for a packet to travel from MN\#1 to MN\#2 can be represented as follows:

$$
T_{\text {ro }}=2 t_{\text {map }}+2 t_{a r}+t_{m 12}+t_{\text {tunnel }}
$$

The advantage of route optimization compared to non-route optimization can be represented as follows:

$$
T_{\text {non_ro }}-T_{\text {ro }}=2 t_{m l}-t_{m 12}+t_{\text {tunnel }}
$$

In general, the distance between the MAG and LMA is considerably greater than the distance between the adjacent MAGs. From the above equation, it is found that route optimization is more effective when the value of $t_{m l}$ (distance between the MAG and LMA) is greater and the value of $t_{m 12}$ (distance between MAG\#1 and MAG\#2) is less. Figure 12 shows the effect of route optimization when the distance between the MAG and LMA is increased given a fixed distance between MAG\#1 and MAG\#2. Figure 13 shows the effect of route optimization when the distance between MAG\#1 and MAG\#2 is increased given a fixed delay between the MAG and LMA. Analyzing Figure 12 and Figure 13, it is evident that route optimization gain becomes more prominent when the distance between the MAG and LMA increases and the distance between MAG\#1 and MAG\#2 decreases. On the other hand, even if the delay between the MAG and LMA is small, route optimization technique can reduce bandwidth consumption.

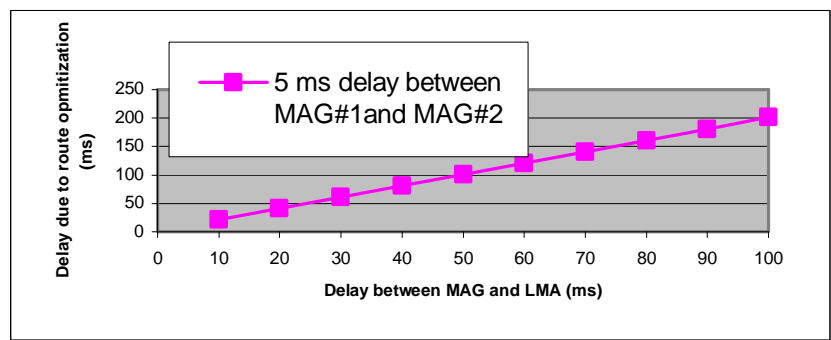

Figure 12: Effect of route optimization (as delay between MAG and LMA varies) 




Figure 13: Effect of route optimization (as delay between MAG\#1 and MAG\#2 varies)

\section{B. Experimental Results}

We have implemented the BCE-based route optimization technique (Approach 1) for PMIPv6 in the IMS (IP Multimedia Subsystem) environment. We discuss the experimental results for both roaming and non-roaming cases.

\section{Experimental results for non-roaming case:}

Authors described some handoff results of a normal PMIPv6 experiment in reference [16]. In this section, we further provide some of the implementation details and experimental results by applying route optimization techniques.

Figure 14 shows the functional components of the nonroaming IMS testbed. In this testbed, there are two visited networks and one home network. All components in the testbed are Linux-based. The home network is equipped with an LMA and the visited networks are equipped with a few MAGs. We applied varying amounts of delay between the MAG and the LMA by using the " $t c$ " (traffic control) utility of Linux so that the distance between the MAG and the LMA could be emulated. In particular, we added a round trip delay of $50 \mathrm{~ms}$, $100 \mathrm{~ms}, 150 \mathrm{~ms}$ and $200 \mathrm{~ms}$ between MAGs and the LMA. We measured the delay associated with the SIP signaling and RTP media.

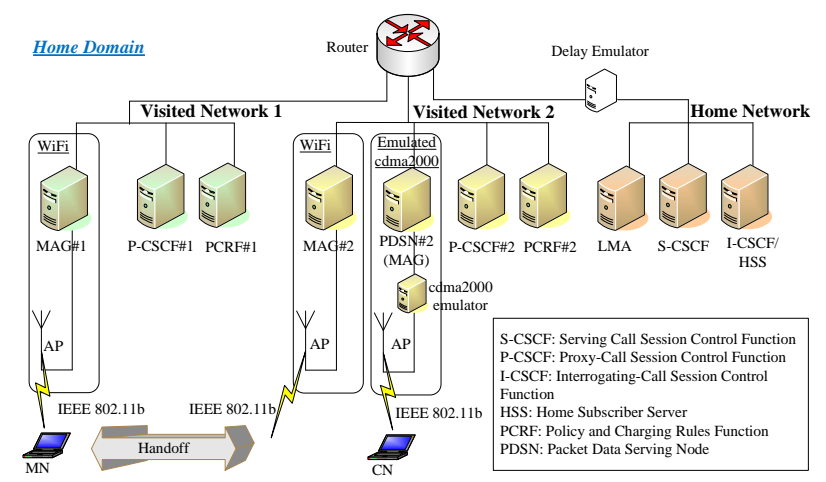

Figure 14: IMS testbed for intra-LMA PMIPv6 route optimization (non-roaming)

We extended PMIPv6 source code that was derived from the MIPv6 source code [17]. An additional thread was added that uses the "nfnetlink_queue" subsystem to retrieve packets from the interface. "ip6tables" is used to route traffic to PMIPv6 running on the LMA. We applied the following ip6tables rule to capture the traffic on a specific port.

ip6tables -A FORWARD -p udp -m mport --ports XXXXX,YYYYY -j QUEUE

When a packet matching the ip6tables rule is found, it is queued for PMIPv6 operation. The "route_optimization" thread receives the packet and checks to see if the source and destination addresses are those handled by this LMA. If so, it checks to see if it has dealt with this pair before. If not, it creates a "ROPTIMIZE BUL" and sends it to both the source and destination MAGs. When the MAGs receive this "ROPTIMIZE" message, they build a tunnel between each other and add appropriate routing entries to use the tunnel.

In order to make the optimization technique lightweight and compatible with the existing binding update messages, a slight extension to the existing PBU method is proposed to facilitate route optimization. We added a new flag " $C$ " to the existing PBU message so that the CBU message could be differentiated from the PBU message. Also, the CBU message in the interLMA scenario needs to include additional addresses such as the source address, destination address and destination MAG address. Thus, a new mobility option type needs to be defined to carry these IP address prefixes (MN-prefix, CN-prefix) and the MAG address. Alternatively, the MAG address may be contained in an alternate care-of-address option. These prefixes may be sent using an HNP (Home Network Prefix) option as well. For brevity, a CBU message format is only shown in Figure 15.

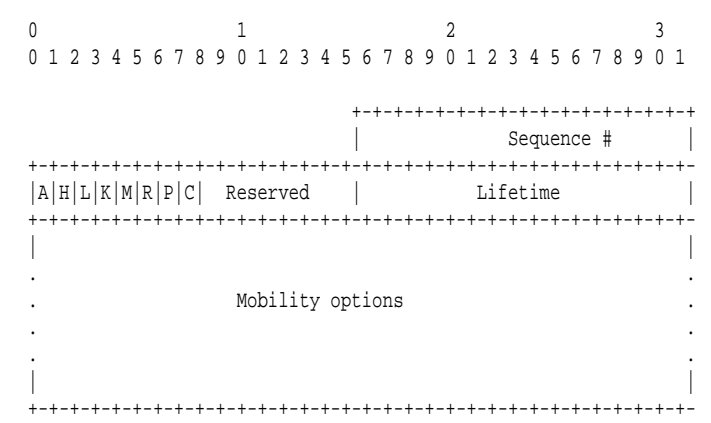

Figure 15: CBU message format

Table 1 compares the results of route optimization and nonroute optimization techniques. In particular, these results include end-to-end media delay, signaling delay between the MNs, handoff delay and packet loss. All the SIP-related signaling such as REGISTER and INVITE traverse all the way to the home network using the tunnels between the MAG and the LMA. Also, in the absence of route optimization, the media traffic flows via the LMA. When route optimization is in place, media traffic bypasses the LMA traversal and flows between the corresponding MAGs over the tunnel that is set up between the MAGs. Thus, the signaling traffic does not benefit from this route optimization technique in an IMS network since all the signaling traffic need to travel all the way to the home network. 
Table 1: Signaling and media delay in non-roaming case

\begin{tabular}{|c|c|c|c|c|c|c|c|c|}
\hline $\begin{array}{c}\text { Added round } \\
\text { trip delay } \\
\text { between } \\
\text { MAG and } \\
\text { LMA }\end{array}$ & \multicolumn{2}{|c|}{$50 \mathrm{~ms}$} & \multicolumn{2}{|c|}{$100 \mathrm{~ms}$} & \multicolumn{2}{|c|}{$150 \mathrm{~ms}$} & \multicolumn{2}{|c|}{$200 \mathrm{~ms}$} \\
\hline $\begin{array}{c}\text { Route } \\
\text { optimization }\end{array}$ & Applied & $\begin{array}{c}\text { Not } \\
\text { applied }\end{array}$ & Applied & $\begin{array}{c}\text { Not } \\
\text { applied }\end{array}$ & Applied & $\begin{array}{c}\text { Not } \\
\text { applied }\end{array}$ & Applied & $\begin{array}{c}\text { Not } \\
\text { applied }\end{array}$ \\
\hline $\begin{array}{c}\text { End-to-end } \\
\text { media delay } \\
\text { (ms) }\end{array}$ & 12 & 71 & 12 & 107 & 15 & 167 & 12 & 213 \\
\hline $\begin{array}{c}\text { Handoff } \\
\text { delay (ms) }\end{array}$ & 10.72 & 10.97 & 11.78 & 11.51 & 12.73 & 12.89 & 13.82 & 14.45 \\
\hline $\begin{array}{c}\text { Packet loss } \\
\text { (packet) }\end{array}$ & 177 & 181 & 193 & 191 & 208 & 214 & 224 & 290 \\
\hline $\begin{array}{c}\text { SIP } \\
\text { REGISTER } \\
\text { delay (s) }\end{array}$ & 2.34 & 2.50 & 2.84 & 2.82 & 3.40 & 3.41 & 3.90 & 4.18 \\
\hline $\begin{array}{c}\text { SIP INVITE } \\
\text { delay (s) }\end{array}$ & 12.82 & 11.00 & 11.75 & 12.00 & 13.25 & 13.49 & 13.83 & 12.91 \\
\hline
\end{tabular}

It is quite evident from Table 1 that end-to-end media delay in route optimization does not vary much even if the delay between the LMA and the MAG is increased. We also evaluate the experimental results for handoff delays and the associated packet loss. In this experiment, we do not apply any layer 2 and layer 3 optimized handoff mechanisms. Some other observations from the experiment are provided below.

- $\quad$ The PMIPv6 route optimization technique only reduces the end-to-end media delay between the $\mathrm{MN}$ and CN. However, this optimization does not help the SIP signaling delay because these signaling messages traverse via the home network.

- The first few packets do not get the benefit of the route optimization process because the first packet itself triggers the route optimization procedure.

- In some cases, if the LMA is far from MAG, there is a possibility of out-of-order packets and jitter between the first few packets that travel via the LMA and subsequent packets that are route optimized. Thus, it could be a possible tradeoff in this approach. However, for real-time streaming traffic, this specific problem is taken care of by a playout buffer at the end system.

- The values of handoff delay and packet loss do not change between route optimization and non-route optimization for any specific LMA or MAG-related delay. In both cases, the $\mathrm{MN}$ is subjected to similar layer 2, layer 3 and signaling delay after the handoff. However, the end-to-end delay for the first packet after the handoff still does not get the benefit from the route optimization procedure. In addition, in an IMS environment, completion of the SIP procedure (SIP REGISTER and SIP Re-INVITE) is a prerequisite before the MAG opens up the firewall to send the traffic through as specified in [18] and [19]. This will delay the first packet after the handoff.

\section{Experimental results for roaming case:}

Figure 16 shows the PMIPv6 route optimization for the roaming case. In this case, there are two PMIPv6 tunnels from the MN that is in the visited domain.

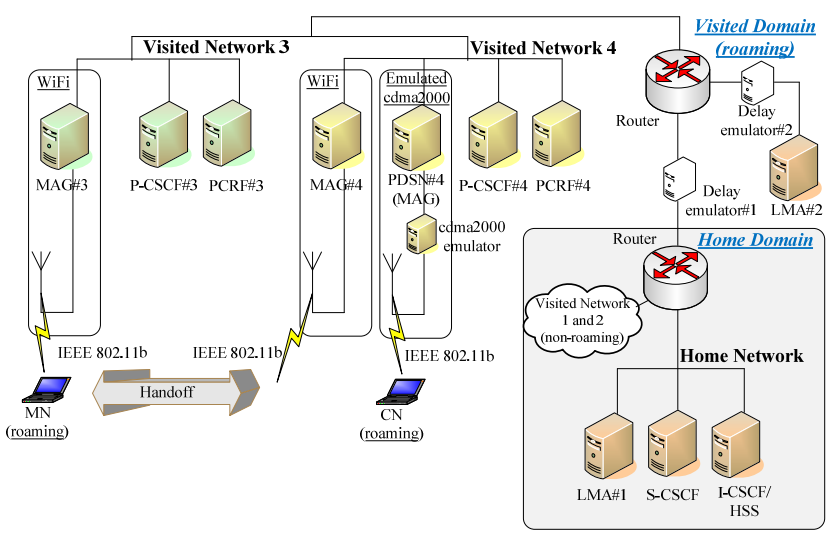

Figure 16: IMS testbed for intra-LMA PMIPv6 route optimization (roaming with multiple tunneling)

The multiple tunneling as described in reference [16] is illustrated in Figure 17. There is one PMIPv6 tunnel between the MAG and LMA in the visited domain and there is another tunnel between the MAG and LMA in the home domain. Both the $\mathrm{MN}$ and $\mathrm{CN}$ are in the visited domain. All the SIP-related signaling messages traverse all the way to the home network in the home domain using the tunnels between the MAG and LMA in the home domain. When route optimization is in place, media traffic bypasses the LMA and flows between the corresponding MAGs in the visited domain. However, the SIP signaling traffic does not get any benefit from this route optimization technique similar to the non-roaming case.

We applied three different amounts of delay between the MAG and LMA in the visited domain and between the MAG and LMA in the home domain and show the results in Table 2. Delays applied between the MAGs and the LMA in the visited network are $25 \mathrm{~ms}, 50 \mathrm{~ms}$ and $100 \mathrm{~ms}$. Similarly, delays applied between the MAGs and the LMA in the home domain are $50 \mathrm{~ms}, 100 \mathrm{~ms}$ and $200 \mathrm{~ms}$. When the route optimization technique is applied, media delay is not affected even if the delay between MAGs and the LMA is increased. Media delay stays about $85 \mathrm{~ms}$. On the other hand, SIP signaling delay is affected quite adversely when the delay between the MAGs and the LMA is increased. Since SIP has an inbuilt retransmission procedure, numerous retransmissions are observed when the delay between the MAGs and the LMA in the home domain is $200 \mathrm{~ms}$, leading to a significant increase in call setup delay. SIP signaling messages are also subjected to redundant routing where the message needs to traverse to a home network before reaching the P-CSCF (Proxy-Call Session Control Function) in the visited network. 


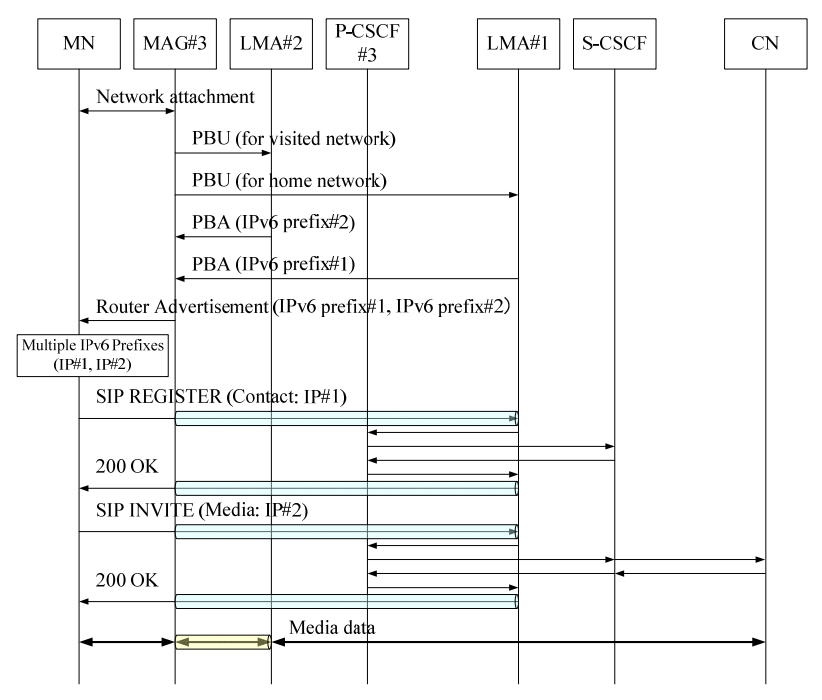

Figure 17: Multiple PMIPv6 tunneling for roaming

We analyze a specific instance when the delay between the MAG and LMA in the visited domain is set to $100 \mathrm{~ms}$. In this case, media delivery takes about $222 \mathrm{~ms}$ between the MN and $\mathrm{CN}$. However, when route optimization is applied, the media delay is about $90 \mathrm{~ms}$. Assuming the routing delay is the same, the additional $132 \mathrm{~ms}$ is due to the added delay of $100 \mathrm{~ms}$ plus the additional tunneling setup delay for one tunnel. Thus, the tunneling delay for one tunnel is $32 \mathrm{~ms}$, and the routing delay is $58 \mathrm{~ms}$. In the case of route optimization, there is only one tunnel (between the MAGs) compared to two tunnels (MAG\#3 and LMA, and MAG\#4 and the LMA) in the non-route optimization case.

Table 2: Signaling and media delay in roaming case

\begin{tabular}{|c|c|c|c|c|c|c|}
\hline \multirow{2}{*}{$\begin{array}{c}\text { Added round trip } \\
\text { delay between MAG } \\
\text { and LMA }\end{array}$} & $\begin{array}{c}\text { MAG and } \\
\text { LMA\#2 }\end{array}$ & $\begin{array}{c}\text { MAG and } \\
\text { LMA\#1 }\end{array}$ & $\begin{array}{c}\text { MAG and } \\
\text { LMA\#2 }\end{array}$ & $\begin{array}{c}\text { MAG and } \\
\text { LMA\#1 }\end{array}$ & $\begin{array}{c}\text { MAG and } \\
\text { LMA\#2 }\end{array}$ & $\begin{array}{c}\text { MAG and } \\
\text { LMA\#1 }\end{array}$ \\
\cline { 2 - 6 } & $25 \mathrm{~ms}$ & $50 \mathrm{~ms}$ & $50 \mathrm{~ms}$ & $100 \mathrm{~ms}$ & $100 \mathrm{~ms}$ & $200 \mathrm{~ms}$ \\
\hline $\begin{array}{c}\text { End-to-end media } \\
\text { delay with route } \\
\text { optimization } \\
\text { (ms) }\end{array}$ & 85 & 82 & 90 \\
\hline $\begin{array}{c}\text { End-to-end media } \\
\text { delay with non-route } \\
\text { optimization } \\
\text { (ms) }\end{array}$ & 102 & 133 & 222 \\
\hline $\begin{array}{c}\text { SIP REGISTER delay } \\
\text { (s) }\end{array}$ & 2.41 & 2.67 & 3.86 \\
\hline SIP INVITE delay (s) & 5.55 & 8.33 & $\begin{array}{c}\text { Unmeasurable level } \\
\text { due to a lot of } \\
\text { retransmission }\end{array}$ \\
\hline
\end{tabular}

\section{CONCLUSIONS}

Although PMIPv6 is designed to address the requirement needed to support intra-domain mobility, route optimization is needed to further reduce the data traversal path between two communicating nodes when these are away from the home network. We proposed several route optimization techniques that can localize packet forwarding between the MAGs instead of the packet getting routed via the LMA. Route optimization becomes more effective as the distance between the MAGs and the LMA increases and the distance between the neighboring MAGs decreases. Experimental results from implementation of binding cache-based approach show that media delay is limited to the sum of the local routing delay and single tunneling delay, and is not affected by the increasing distance between the MAG and LMA. Thus, local media forwarding using route optimization technique is an essential enhancement to PMIPv6 that can support real time communication.

\section{REFERENCES}

[1] Ramjee et al., "Hawaii: A Domain-based Approach for Supporting Mobility in Wide-area,” ICNP'99, Nov. 1999.

[2] A. Campbell, J. Gomez-Castellanos, "IP Micro-mobility Protocols," vol 4, Oct. 2004.

[3] H. Soliman et al. "Hierarchical Mobile IPv6 Mobility Management (HMIPv6)," IETF RFC 4140, Aug. 2005.

[4] J. Kempf, "Goals for Network-Based Localized Mobility Management (NETLMM)," RFC 4831, Apr. 2007.

[5] J. Kempf, "Problem Statement for Network-Based Localized Mobility Management (NETLMM)," IETF RFC 4830, Apr. 2007.

[6] S. Gundavelli et al., "Proxy Mobile IPv6," IETF RFC 5213, Aug. 2008.

[7] D. Johnson et al., "Mobility Support in IPv6," IETF RFC 3775, June 2004.

[8] C. Perkins, “IP Mobility Support for IPv4,” IETF RFC 3344, Aug. 2002.

[9] T. Chiba et al., "Trombone Routing Mitigation techniques for IMS/MMD Networks,” IEEE WCNC 2007, Mar. 2007.

[10] S. Jeong at al, "Problem Statement and Requirements for Route Optimization in PMIPv6," IETF draft-jeongnetlmm-pmipv6-roeq-01, Nov. 2007.

[11]B. Sarikaya et al., "PMIPv6 Route Optimization Protocol," IETF draft-qin-netlmm-pmipro-00,”Feb. 2008.

[12] M. Liebsch et al., "Route Optimization for Proxy Mobile IPv6," IETF draft-abeille-netlmm-proxymip6ro-01, Nov. 2007.

[13] R. Koodli et al., "Local Forwarding in Proxy Mobile IPv6," IETF draft-koodli-netlmm-local-forwarding-00, July 2008.

[14] H. Yokota, T. Kubo, A. Idoue, M. Inoue and K. Mahmud, "Decentralized Micro-Mobility Management for LargeScale Mobile Networks," WPMC 2003, Vol.2, pp.352356, Oct. 2003.

[15] Ki-Sik et al., "Mobility Management for All-IP Mobile Networks: Mobile IPv6 vs. Proxy Mobile IPv6," IEEE Wireless Communications, Apr. 2008.

[16] T. Chiba et al., "Performance Analysis of Next Generation Mobility Protocols for IMS/MMD Networks," IEEE IWCMC 2008, Aug. 2008.

[17] Mobile IPv6, http://www.mobile-ipv6.org/.

[18]3GPP," "Policy and charging control over Gx reference point," TS 29.212, v8.0.0, June 2008.

[19]3GPP," "Policy and charging control over Rx reference point," TS 29.214, v8.1.0, June 2008. 